\title{
Development of a system for the assessment of a dual-task performance based on a motion- capture device
}

\section{$\operatorname{AUTHOR}(\mathrm{S})$ :}

Okamoto, Kazuya; Kayama, Hiroki; Yamada, Minoru; Kume, Naoto; Kuroda, Tomohiro; Aoyama, Tomoki

\section{CITATION:}

Okamoto, Kazuya ... [et al]. Development of a system for the assessment of a dual-task performance based on a motion-capture device. International Journal on Disability and Human Development 2014, 13(3): 333-336

\section{ISSUE DATE:}

\section{4-08-13}

\section{URL:}

http://hdl.handle.net/2433/198570

\section{RIGHT:}

C 2014 by De Gruyter; 許諾条件により本文ファイルは2015--08-13に公 開. 


\section{Development of a system for the assessment of a dual-task performance based on a motion-capture device}

\begin{abstract}
The authors produced a dual task (DT) that provided a dynamic balance task and a cognitive task in a game system using motion sensors and virtual images. There had been no DT where a cognitive task needs a dynamic balance task that requires full-body motions. We developed and evaluated a game system to assess the performance of the DT. The DT was to solve a Sudoku puzzle using full-body motions like Tái Chi. An ability to perform a DT is intimately related to risk of falls. To evaluate the developed system, we compared the performance of elderly people and young people. Generally, elderly people are at a higher risk of falls. Twenty elderly community-dwelling adults (mean age, $73.0 \pm 6.2$ years) and 16 young adults (mean age, $21.8 \pm 1.0$ years) participated in this study. To compare the two groups, we applied an independent-samples t-test. The time taken for the elderly people was $60.6 \pm 43.2 \mathrm{~s}$, whereas the time taken for the young people was $16.0 \pm 4.8 \mathrm{~s}$. The difference is statistically significant $(p<0.05)$. This result suggests that the developed game system is useful for the evaluation of the DT performance.
\end{abstract}

Keywords: balance task; cognitive task; dual task; elderly community-dwelling adults; evaluation of systems; risk of falls.

DOI 10.1515/ijdhd-2014-0324

Received April 10, 2013; accepted May 23, 2013; previously published online August 13, 2014

*Corresponding author: Kazuya Okamoto, PhD, Assistant Professor, Kyoto University Hospital, Division of Medical Information Technology and Administration Planning, 54 Kawaharacho, Shogoin, Sakyo-ku, Kyoto-shi, Kyoto, Japan,

E-mail: kazuya@kuhp.kyoto-u.ac.jp

Naoto Kume and Tomohiro Kuroda: Division of Medical Information Technology and Administration Planning, Kyoto University Hospital, Japan

Hiroki Kayama, Minoru Yamada and Tomoki Aoyama: Department of Physical Therapy, Human Health Sciences, Graduate School of Medicine, Kyoto University, Japan

\section{Introduction}

Falls are fairly common among elderly people. Blake et al. showed that $35 \%$ of elderly people aged 65 years and older reported one or more falls in a year [1]. Load and Ward pointed out that people beyond age 65 increase sway areas because their reduced vision is less able to supplement peripheral input than younger people [2]. Because falls of elderly people often result in fractures, it is important to predict these falls and prevent them.

It is widely recognized that balance disorders of elderly people are related to multiple impairments, such as weakness and cognitive impairments [3]. Lundin-Olsson et al. reported a novel method for risk analysis of falls based on dual-task (DT) performance [4]. DT can consist of a combination of a dynamic balance task and a cognitive task. An example of an effective dynamic balance task is Tái Chi, which is based on full-body motions. Leung et al. demonstrated that Tái Chi reduces falls in the non-frail elderly [5]. Also, an example of an effective cognitive task is Sudoku. Sudoku is a logicbased puzzle known familiarly throughout the world. Nombela et al. showed that cognitive training based on Sudoku exercises improves the cognitive performance of Parkinson's patients in the Stroop test [6]. Although each performance of a dynamic balance task or cognitive task can be assessed easily, it is more difficult to assess DT performance involving two tasks. Yamada et al. considered that the evaluation of the performance of the secondary task is insufficient in the previous research using DT protocols [7]. For assessing DT ability, they developed a smartphone-based application where a player is required concurrently to walk and to roll a virtual ball on a smartphone using his or her wrist. However, there has been no DT where a cognitive task needs a dynamic balance task that requires full-body motions. In this study, we developed a DT where a cognitive task needs a dynamic balance task that requires full-body motions. 
We used Sudoku and Tái Chi as a cognitive task and a dynamic balance task, respectively. Suppose that we made large Sudoku models using physically large grids and blocks to require full-body motions. A player solving the Sudoku would have difficulty in recognizing the large instance, because they must be near the instance to solve it. Hence, we projected a player to a figure on a screen and superimposed an instance of Sudoku on the same screen. When the instance was large compared to the figure on the screen, a player was required to use full-body motions. Also, because there was an appropriate distance between the player and the screen, he or she could recognize the instance.

We produced a DT that provided a dynamic balance task and a cognitive task at the same time in a game system using motion sensors and virtual images. The DT was to solve Sudoku using full-body motions like Tái Chi. We also developed a system to assess the performance of the DT and evaluate the proposed system.

\section{Methods}

Characteristic full-body motions of Tái Chi included spreading the hands wide, bringing them close, spreading the legs wide, bringing them close, or combinations of these. A subject was asked to do these characteristic full-body motions, which were then navigated to solve Sudoku; in other words, the subject was given an instance of Sudoku and asked to put digits into the vacant boxes provided using the fullbody actions. We produced a DT that provided a dynamic balance task and a cognitive task at the same time in a game system using motion sensors and virtual images. Figure 1 depicts the diagram of the DT and the system that was developed.

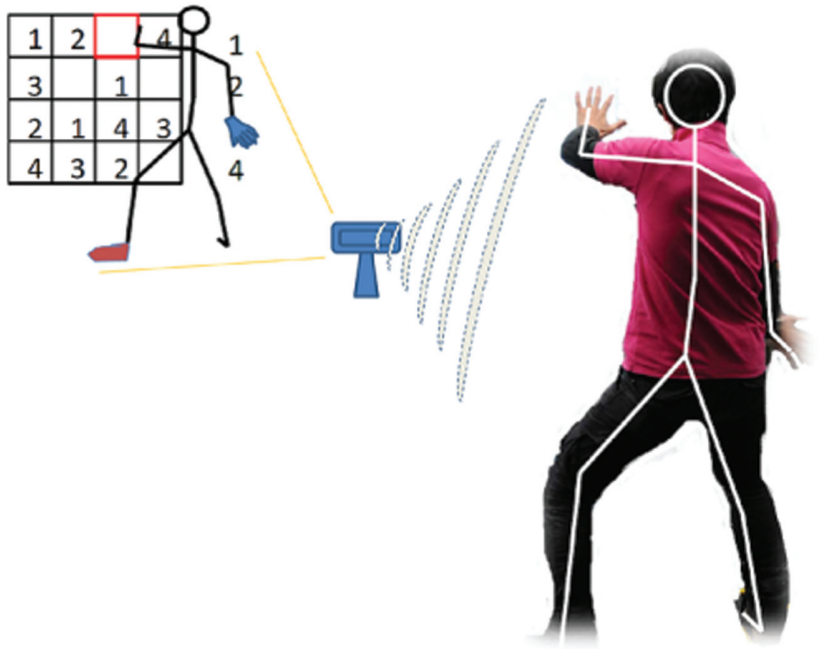

Figure 1 A conceptual diagram of the DT and the developed system.

\section{System configuration and system operation}

The game system used to provide the DT with the assessment of the DT performance consisted of a motion-capture device, a computer, a screen and a projector. A Kinect (Microsoft Co., Redmond, WA, USA) was used as the motion-capture device. Figure 2 depicts the configuration of the game system. We described the system operation according to Figure 3, which depicts the flow chart of the game system.

First, the initialization module prepares a rectangular workspace in the computer's memory. The initialization module loads a Sudoku instance and lays out the instance on the workspace. The Sudoku instance consists of a $4 \times 4$ grid ( 16 boxes). Thirteen boxes were initially filled with digits from 1 to 4 . Many Sudoku instances were prepared in advance. The initialization module chose one of them at random.

The game system uses Kinect as a motion-capture device. The equipment has an infrared projector and an infrared camera. The resolution of the infrared camera was QVGA $(320 \times 240)$, and the horizontal and vertical fields of view were 57 degrees and 43 degrees,

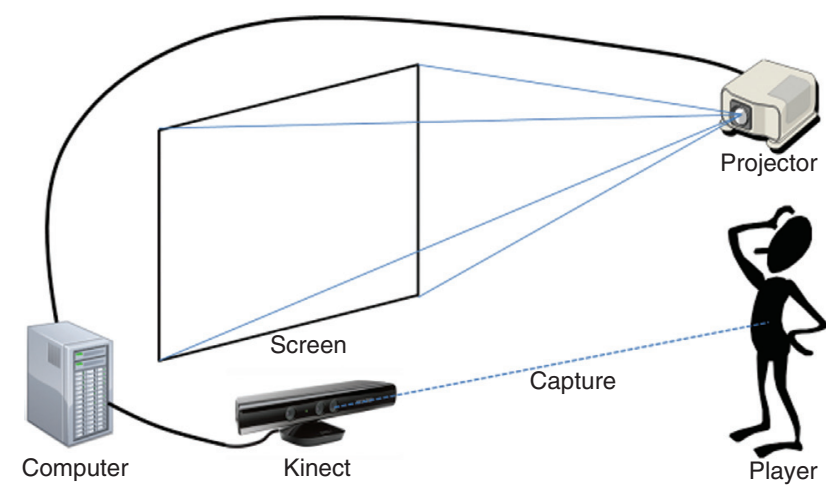

Figure 2 A configuration of the game system.

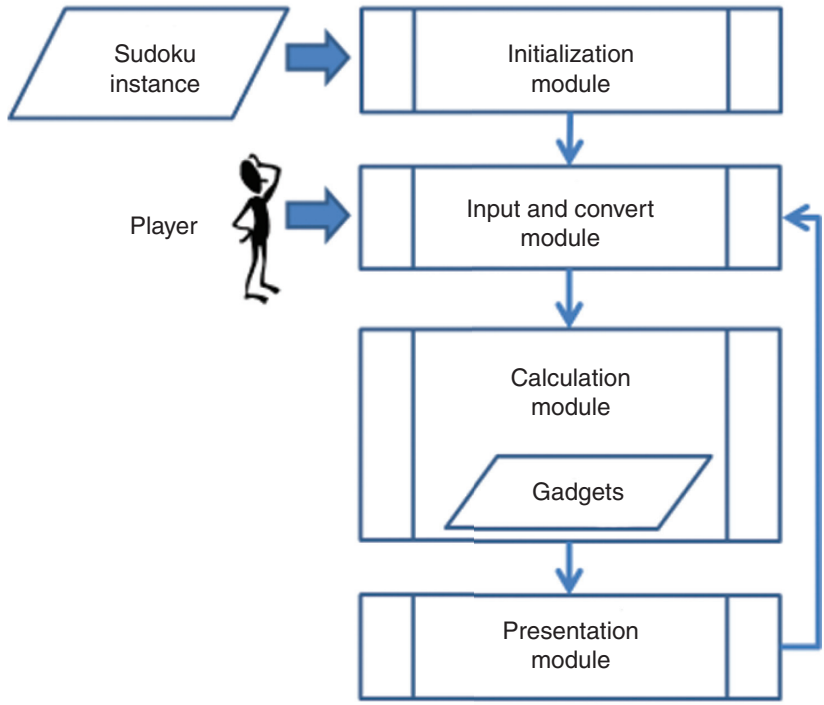

Figure 3 A flow chart of the game system. 


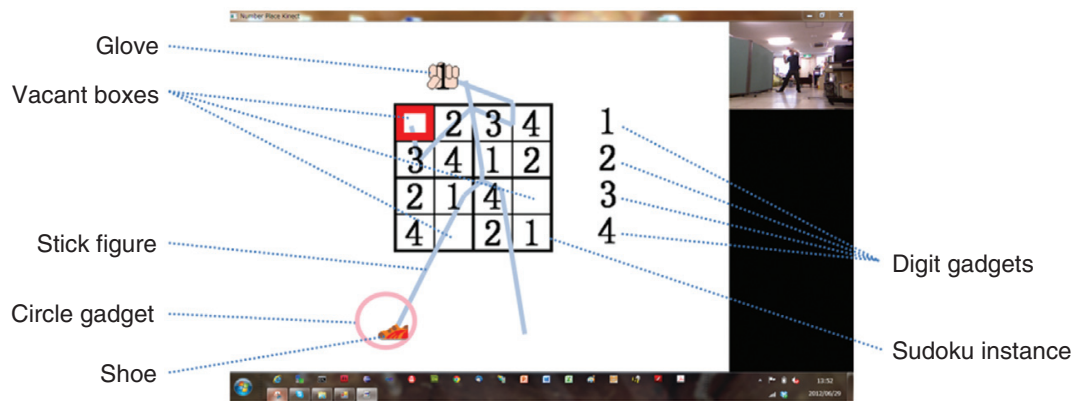

Figure 4 A display of the game system on a screen.

respectively. The camera could detect physical objects in a distance range between $1.2 \mathrm{~m}$ to $3.5 \mathrm{~m}$, and it expressed the depth per each pixel using a 16-bit mono image. The frame rate of the camera was 30 frames per second. An input module received the physical object data by utilizing the Kinect and Kinect for Windows SDK Beta 1. The information included the position and the shape of a player. A conversion module extracted the physical object data related to the player, converted the data to skeleton information, including positional information of joints, and laid out the skeleton information on the workspace in the computer's memory.

The puzzle digits (from 1 to 4 ) and a circle were positioned as far as the right hand joint and the left foot joint of the skeleton could reach, respectively. (Note that the game system supposes that the player stands directly in front of Kinect.) The system determined if the following conditions were satisfied: i) the right hand joint overlaps one of the digit gadgets; ii) the left foot joint overlaps the circle gadget; and iii) both the right hand joint and the left hand joint overlap one of the vacant boxes in the grid of the Sudoku instance (see Figure 4). When both $i$ and ii are satisfied, the player grabbed a corresponding digit. When the player had a digit and both ii and iii were satisfied, he or she tried to fill the vacant box using the digit. When the digit was correct, depending on the rules of Sudoku, the vacant box became filled.

A presentation module presents the Sudoku instance, the skeleton information, and the gadgets on a screen. The skeleton information was shown as a stick figure. The images of a glove and a shoe were superimposed on the right hand joint and the left foot joint so that the player could recognize them easily. In addition, when all the vacant boxes were filled, the time taken to fill them was measured and displayed. The display size could be changed freely. Figure 4 depicts the display of the game system.

\section{Game scenario}

The player was asked to put digits into the remaining three vacant boxes. First, the player was requested to select a digit to fill vacant boxes. Digit selection required that the player spread his or her hands and legs. Next, to put the selected digit into a vacant box, the player was requested to select the vacant box. The player must move the right hand close to the left hand. Concurrently, because the player must follow the rules of Sudoku, the game required cognitive skill. The player finished the DT game when he or she filled all three vacant boxes. The system measured the time taken to solve the DT.

\section{Evaluation}

To evaluate the developed system, we compared the performance of elderly people and young people. Generally, elderly people are at a higher risk of falls. Twenty elderly community-dwelling adults (mean age, $73.0 \pm 6.2$ years) and 16 young adults (mean age, $21.8 \pm 1.0$ year) participated in this study. To compare the two groups, we applied an independent-samples t-test, where $\mathrm{p}<0.05$ was considered statistically significant.

\section{Results}

Figure 5 depicts the result of the evaluation. The time taken for the elderly people was $60.6 \pm 43.2 \mathrm{~s}$ while the time taken for the young people was $16.0 \pm 4.8 \mathrm{~s}$. The difference is statistically significant $(\mathrm{p}<0.05)$ although the variability reported for the elderly group was large. This result suggests that the developed game system is useful for the evaluation of the DT performance.

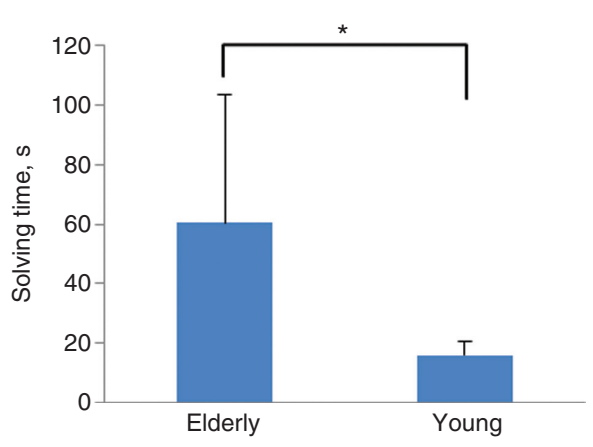

Figure 5 A comparison between elderly and young people of the time taken to solve the DT. Statistical significance $\left({ }^{\star} p<0.05\right)$. 


\section{Discussion}

Because the difference of time taken to solve the DT for elderly people and young people, the game system may be able to assess risk of falls. We will continue to evaluate the system by comparing with single tasks of the DT and other physical performance tests that are widely used to identify high-risk elderly adults.

In the experiments, almost all the subjects said that they wanted to try the game again, that is, they seemed to feel the DT interesting. If the game is sufficiently interesting for players to continue playing the game, we can evaluate whether the game system can be used as a training device.

Negative feedback of the subjects was not given in the experiments. This may mean that the subjects were performing the movements as expected, and that the system could obtain skeleton information including positional information of joints to succeed at the DT game.

Acknowledgments: This work was supported by KAKENHI (24700248).

\section{References}

1. Blake AJ, Morgan K, Bendall MJ, Dallosso H, Ebrahim SB, Arie TH, et al. Falls by elderly people at home: prevalence and associated factors. Age Ageing 1988;17:365-72.

2. Lord SR, Ward JA. Age-associated differences in sensori-motor function and balance in community dwelling women. Age Ageing 1994:23:452-460.

3. Tinetti ME, Inouye SK, Gill TM, Doucette JT. Shared risk factors for falls, incontinence and functional dependence: unifying the approach to geriatric syndromes. J Am Med Assoc 1995;273:1348-53.

4. Lundin-Olsson L, Nyberg L, Gustafson Y. Attention, frailty, and falls: the effect of a manual task on basic mobility. Proc IEEE Intl Conf Autom Control 1998;46:231-45.

5. Leung DP, Chan CK, Tsang HW, Tsang WW, Jones AY. Tai Chi as an intervention to improve balance and reduce falls in older adults: a systematic and meta-analytical review. Altern Ther Health Med 2011;17:40-8.

6. Nombela C, Bustillo PJ, Castell PF, Sanchez L, Medina V, Herrero MT. Cognitive rehabilitation in Parkinson's disease: evidence from neuroimaging. Front Neurol 2011;2:82.

7. Yamada M, Aoyama T, Okamoto K, Nagai K, Tanaka B, Takemura T. Using a Smartphone while walking: a measure of dual-tasking ability as a falls risk assessment tool. Age Ageing 2011;40:516-9. 